\title{
Phonon Scattering at Siliconcrystal Surfaces
}

\author{
D. Marx * and W. Eisenmenger \\ Physikalisches Institut der Universität, Stuttgart, \\ Federal Republic of Germany
}

Received July 16, 1982

\begin{abstract}
Our observations of the reflection or backscattering of high-frequency phonons $(v$ $=280 \mathrm{GHz}$ to $1 \mathrm{THz}$ ) at silicon-solid interfaces disagree significantly with predictions from the acoustic mismatch model. Interfaces composed of materials theoretically wellmatched, show high scattering experimentally. In contrast, interfaces theoretically poorly matched, show less phonon scattering than expected. Generally, this is best expressed by the fact that the interface scattering ranges from roughly $30-60 \%$ for different phonon modes with little dependence on the material covering the silicon crystal and different techniques of interface preparations. Thus, our experiments indicate that the well-known Kapitza anomaly of the phonon scattering at solid-liquid helium interfaces is not a special case; the same anomaly appears to be present at all tested interfaces. Our experiments are compared with detailed calculations which either assume pure specular or pure diffusive scattering. In these calculations the influence of the crystal anisotropy for the phonon propagation (phonon focussing) is included. This comparison shows, especially for the free silicon surface, that phonons are completely diffuse scattered. Hence, the acoustic mismatched model relying on specular reflection cannot be applied to the real silicon interface.

The frequency dependence of phonon scattering at a free silicon interface indicates the existence of at least two different diffusive scattering mechanisms. Within our experimental limits in these two scattering processes the phonons are elastically scattered.
\end{abstract}

\section{Introduction}

In general, the interface between two different materials represents a heat-flux resistance. This thermal boundary resistance was first discussed by Kapitza [1] for the copper/helium interface (Kapitza resistance).

If the two materials are insulators, the boundary resistance is mainly due to the scattering of phonons at the interface. Little [2] calculated the thermal boundary resistance caused by the scattering of phonons within the frame of the continuum theory. This so-called acoustic mismatch theory describes the boundary resistance between solids measured in heat conduction experiments fairly well [4-8], but fails at interfaces between solids and helium in the temperature range above $0.1 \mathrm{~K}$. In recent years, a lot of

* Present address: Porsche AG, D-7251 Weissach, FRG work has been done on this so-called Kapitza anomaly at the solid-helium interface $[9,10]$.

In contrast to heat conduction experiments, measurements with superconducting tunneling junctions as phonon generators and detectors [11] have the advantage of frequency selectivity. Using this technique Trumpp and Eisenmenger [12] found that the transmission of quasimonochromatic acoustic phonons (frequency: $280 \mathrm{GHz}$ ) through metal-insulator interfaces is about $30 \%$ of the value predicted by the acoustic mismatch theory. This gives rise to a considerable reduction of the sensitivity in phonon spectroscopy experiments compared with the expectations.

For the phonon spectroscopy with superconducting tunneling junctions it is an important question whether the discrepancy between theory and experi- 
ment is of principal nature or caused by the special preparation of the interfaces.

In the present work we studied the phonon scattering at silicon/metal interfaces in reflection experiments with phonon pulses and compared with the acoustic mismatch theory. Reflection experiments with phonon pulses allow mode and frequency selection. Furthermore, as a main advantage, reflection or backscattering experiments give the possibility of preparing the studied interface without influencing the generator and detector preparation. Therefore, the parameters of the interface can be varied in a wide range.

The phonon scattering at the interfaces is compared with the scattering at a free surface. At interfaces between substrate and condensed gases these comparative measurements can be made with one generator/detector pair. Without having the possibility of evaporation in the measuring cryostate we use for the reflection experiments at metal-substrate interfaces two independent generator/detector pairs. One generator/detector pair serves for reference measurement of the phonon scattering at the free substrate surface, whereas the other generator/detector pair probes the scattering at the metal/substrate interface. As phonon generators, we use mostly constantan heaters, as detectors superconducting tin tunneling junctions. For a more detailed frequency analysis, we use tin tunneling junctions as generators and tin or aluminium tunneling junctions as detectors.

\section{Physical Basis}

\subsection{Phonon Focusing}

The silicon substrate with cubic symmetry exhibits elastic anisotropy, i.e. the phase velocity of a plane wave in the substrate depends on the direction of the $\mathbf{k}$-vector. Figure 1 shows cross-sections of the corresponding slowness surfaces of plane waves in silicon [14]. Since the group and phase velocities in general are not parallel, the phonon flux from an incoherent phonon generator, emitting isotropically into the $\mathbf{k}$-space is anisotropic in the $\mathbf{v}$-space. This effect is well-known as phonon focusing $[15,16]$. Figure 2 shows the calculated distribution of the energy density at the substrate surface opposite to the generator. In this calculation equations of Every [17] for cubic materials have been used, yielding the phase and group velocities and the directions of the displacements for plane waves with a definite $k$ vector. The corresponding energy density distributions have been experimentally verified by Eisenmenger [18] and Wolfe and Northrop [19].
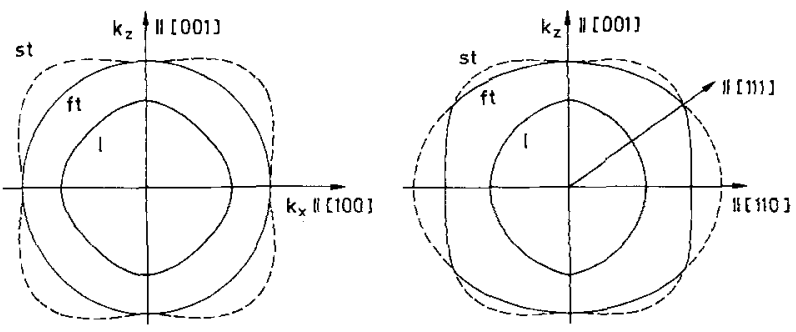

Fig. 1. Cuts through the reciprocal phase velocity surfaces for elastic plane waves with fixed frequency in silicon. $l$ : longitudinal, $f t$ : fast, and st: slow transverse waves. In isotropic material the surfaces are spherical

\subsection{Phonon Reflection at Boundaries}

In calculating the reflection and transmission of phonons at an interface it is assumed the solids to be continuous and the interface to be ideally flat and negligibly thick. Possible contributions of electrons are not considered [4]. So far, there exists no generally accepted theory accounting for the microscopic structure of the interface and the solids. Steinbrïchel [20] has carried out interesting calculations for modified boundary conditions but these do not apply to our experimental situation.

For the scattering of a plane elastic wave at an interface, Snell's law and Fresnel's equations [14] are applied making use of the continuity of the particle velocity $\mathbf{A}$ and the boundary stress. Snell's law contains the conservation of $k$-parallel and specifies the directions of the $\mathbf{k}$-vectors of the scattered waves. The frequencies of the scattered waves are the same as those of the incident wave, since anharmonic processes are not taken into account.

The important reflection coefficients (i.e. the amplitudes of the scattered waves divided by the amplitudes of the incident waves) are listed in the following:

Reflection Coefficient for Incident Fast Transverse Wave. The polarization is horizontal and the reflection coefficient [14] for the experimentally dominant fast transverse mode reads as follows:

$\Gamma_{f t}^{\text {eovered }}=\left|\frac{B}{A}\right|^{2}=\left(\frac{Z_{f t} \cos \theta_{f t}^{\prime}-Z_{f t}^{\prime} \cos \theta_{f t}^{\prime}}{Z_{f t} \cos \theta_{f t}^{\prime}+Z_{f t}^{\prime} \cos \theta_{f t}^{\prime}}\right)^{2}$

with

$\sin \theta_{f t} / \sin \theta_{f t}^{\prime}=\mathrm{c}_{f t} \mid \mathrm{c}_{f t}^{\prime}$

and the acoustic impedances:

$Z_{f t}=\rho c_{f t}$

( $\rho$ : mass density, $c_{f t}$ : phase velocity, see Fig. 3): At the free surface we obtain the reflection coefficient 
$\Gamma_{f t}^{\mathrm{free}}=1$

because no mode conversion occurs.

Reflection Coefficient for Incident Longitudinal Wave. In this case, longitudinal waves, as well as slow transverse waves are excited in reflection and transmission (see Fig. 4). The reflection coefficient for longitudinal phonons is [14]:

$\Gamma_{l l}^{\text {covered }}=\left|\frac{B_{e}}{A_{e}}\right|^{2}=\left|\frac{A_{l l}}{\Delta}\right|^{2}$

with

$\Delta_{l l}=\left|\begin{array}{cccc}-\cos \theta_{l} & -\cos \theta_{l}^{\prime} & \sin \theta_{s t} & \sin \theta_{s t}^{\prime} \\ \sin \theta_{l} & \sin \theta_{l}^{\prime} & -\cos \theta_{s t} & \cos \theta_{s t}^{\prime} \\ \rho c_{l} \cos 2 \theta_{s t} & \rho^{\prime} c_{l}^{\prime} \cos \theta_{s t}^{\prime} & \rho c_{s t} \sin 2 \theta_{s t} & -\rho^{\prime} c_{s t}^{\prime} \sin 2 \theta_{s t}^{\prime} \\ -\rho \frac{c_{s t}^{2}}{c_{l}} \sin 2 \theta_{l} & -\rho^{\prime} \frac{c_{s t}^{\prime 2}}{c_{l}^{\prime}} \sin 2 \theta_{l}^{\prime} & -\rho c_{s t} \cos 2 \theta_{s t} & -\rho^{\prime} c_{s t}^{\prime} \cos 2 \theta_{s t}^{\prime}\end{array}\right|$

and

$\Delta=\left|\begin{array}{cccc}-\cos \theta_{l} & -\cos \theta_{l}^{\prime} & \sin \theta_{s t} & \sin \theta_{s t}^{\prime} \\ -\sin \theta_{l} & \sin \theta_{l}^{\prime} & -\cos \theta_{s t} & \cos \theta_{s t}^{\prime} \\ -\rho c_{l} \cos 2 \theta_{s t} & \rho^{\prime} c_{l}^{\prime} \cos \theta_{s t}^{\prime} & \rho c_{s t} \sin 2 \theta_{s t} & -\rho^{\prime} c_{s t}^{\prime} \sin 2 \theta_{s t}^{\prime} \\ -\rho \frac{c_{s t}^{2}}{c_{l}} \sin 2 \theta_{l} & -\rho^{\prime} \frac{c_{s t}^{\prime 2}}{c_{l}^{\prime}} 2 \theta_{l}^{\prime} & -\rho c_{s t} \cos 2 \theta_{s t} & -\rho^{\prime} c_{s t}^{\prime} \cos 2 \theta_{s t}^{\prime}\end{array}\right|$

and

$\sin \theta_{l} / \sin \theta_{s t}=c_{l} / c_{s t}$

$\sin \theta_{l} / \sin \theta_{l}^{\prime}=c_{l} / c_{l}^{\prime}$

$\sin \theta_{l} / \sin \theta_{s t}^{\prime}=c_{l} / c_{s t}^{\prime}$.

At the free surface (3) reduces to:

$\Gamma_{l l}^{\mathrm{free}}=\left|\frac{B_{l}}{A_{l}}\right|^{2}$

$=\left(\frac{\sin 2 \theta_{s t} \sin 2 \theta_{l}-\left(\frac{c_{l}}{c_{s t}}\right)^{2} \cos ^{2} 2 \theta_{s t}}{\sin 2 \theta_{s t} \sin 2 \theta_{l}+\left(\frac{c_{l}}{c_{s t}}\right)^{2} \cos ^{2} 2 \theta_{s t}}\right)^{2}$.

In our experimental arrangement, specularly reflected phonons without mode conversion travel along $\langle 110\rangle$ directions. In these directions slow transverse phonons are defocused and cannot be detected. Therefore, the corresponding equations are not presented here.

In our experiments the difference in the signal amplitude by phonons backscattered from the free and the covered surface and not the absolute value of the reflection coefficients have been determined.
Thus, we define the experimental reflection factors $R_{\alpha}$ :

$R_{f t}=\Gamma_{f t}^{\text {covered }} / \Gamma_{f t}^{\mathrm{free}}$

and

$R_{l}=\Gamma_{l l}^{\mathrm{covered}} / \Gamma_{l l}^{\mathrm{free}}$.

Several calculated experimental reflection factors and the material constants used are listed in Table 1. These data show that most metals appear to be acoustically well matched to a silicon substrate 

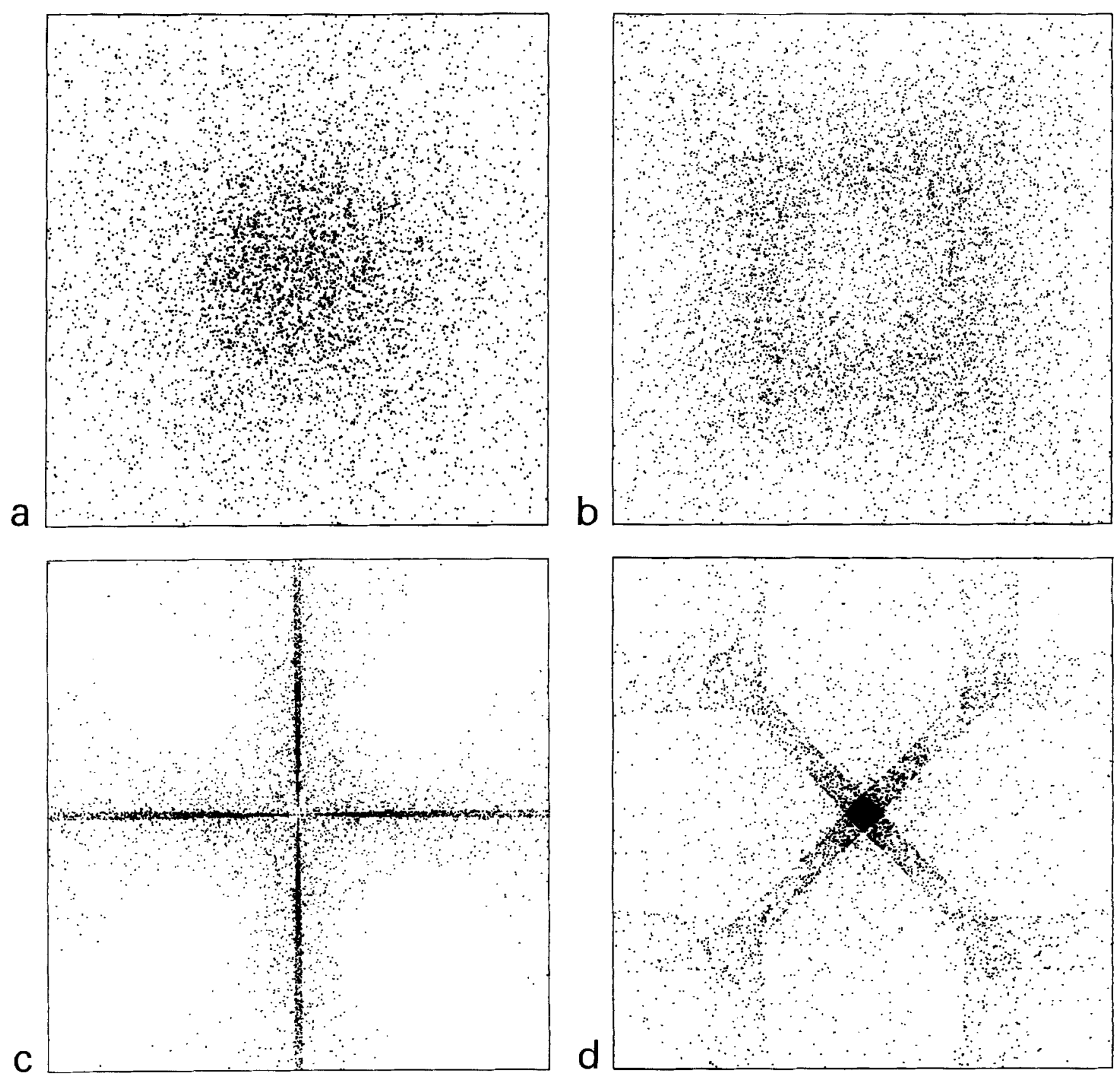

Fig. 2a-d. Calculated distributions of the energy on the substrate surface opposite to the point generator. The ratio of the edges of the substrate is $1: 4: 4$, the sides parallel to $\langle 100\rangle$-directions. Figure $\mathrm{c}$ shows clearly the strong focusing of the fast transverse phonons in the (100)-planes. a Isotropic material. b Silicon, longitudinal phonons. c Silicon, fast transverse phonons. d Silicon, slow transverse phonons

measurements of the frequency dependence. For details regarding the superconducting tunneling junctions as phonon generator and detector see the articles of Eisenmenger [11], and Trumpp and Eisenmenger [12].

The phonon backscattering is measured by a comparative method and evaluated in terms of the experimental reflection factors. By this technique the eventually critical generator and detector parameters are equal and, therefore, do not influence the measured reflection factors.

\section{Experimental Procedure}

Figure 5 shows the arrangement of our reflection experiments schematically. The substrate is pressed with the scattering surface against the rim of the 


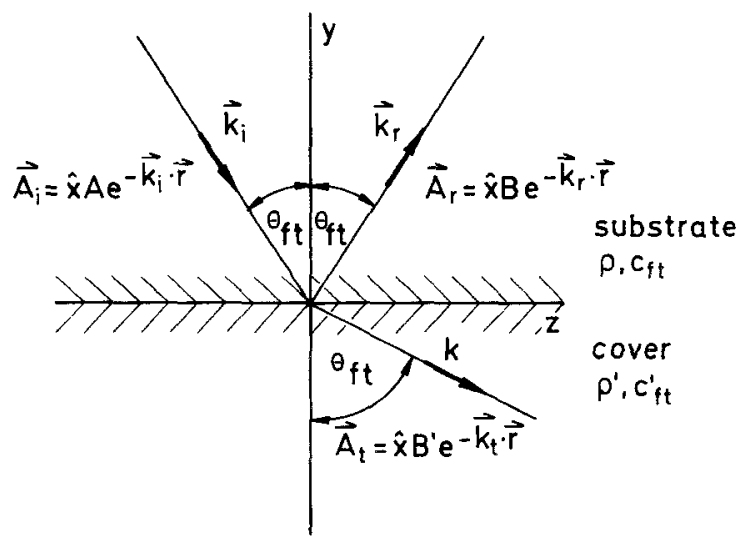

Fig. 3. Reflection and transmission of a plane transverse wave (polarization perpendicular to the incident plane) at a flat interface

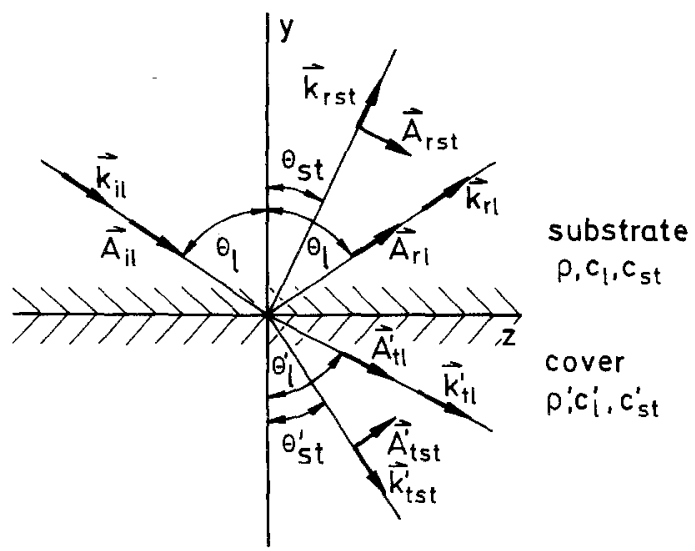

Fig. 4. Reflection and transmission of a plane longitudinal wave at a flat interface. The particle velocities are:

$\mathbf{A}_{i l}=\frac{\mathbf{k}_{i l}}{k_{l}} A_{l} \exp \left\{-i \mathbf{k}_{i l} \cdot \mathbf{r}\right\}$

$\mathbf{A}_{r s t}=\frac{\left\{\hat{x} \times \mathbf{k}_{r s t}\right\}}{\mathbf{k}_{s t}} B_{s t} \exp \left\{-i \mathbf{k}_{r s t} \cdot \mathbf{r}\right\}$

$\mathbf{A}_{r l}=\frac{\mathbf{k}_{r l}}{\mathbf{k}_{l}}=B_{l} \exp \left\{-i \mathbf{k}_{r l} \cdot \mathbf{r}\right\}$

$\mathbf{A}_{t l}^{\prime}=\frac{\mathbf{k}_{i l}}{\mathbf{k}_{l}^{\prime}} B_{l^{\prime}} \exp \left\{-i \mathbf{k}_{t l}^{\prime} \cdot \mathbf{r}\right\}$

$\mathbf{A}_{t s t}^{\prime}=\frac{\left\{\hat{x} \times \mathbf{k}_{t s t}\right\}}{-\mathbf{k}_{s t}^{\prime}} B_{s t}^{\prime} \exp \left\{-i \mathbf{k}_{t s t}^{\prime} \cdot \mathbf{r}\right\}$

vacuum chamber aperture and sealed with indium. The vacuum chamber is evacuated by an ion pump. Phonon generators and detectors are exposed to liquid helium. As substrates we use pure $n$-conducting silicon monocrystals (240 $0 . \mathrm{cm}$, Wacker Chemie) with dimensions $25 \mathrm{~mm} \times 25 \mathrm{~mm} \times 5 \mathrm{~mm}$. Specularly reflected phonons without mode conversion are propagating along $|110|$ directions.
Table 1. Material constants and reflection constants calculated according to (5) and (6). Dates of silicon: $\rho=2.33 \mathrm{~g} / \mathrm{cm}^{3}$, $c_{l}=9 \cdot 19 \cdot 10^{5} \mathrm{~cm} / \mathrm{s}, \quad c_{f t}=5 \cdot 87 \cdot 10^{5} \mathrm{~cm} / \mathrm{s}, \quad c_{s t}=4 \cdot 89 \cdot 10^{5} \mathrm{~cm} / \mathrm{s}$, $\theta=45^{\circ}$. The covering material is assumed to be isotropic

\begin{tabular}{lllllll}
\hline Mat. & $\begin{array}{l}\rho \\
{\left[\mathrm{g} / \mathrm{cm}^{3}\right]}\end{array}$ & $\begin{array}{l}C_{i} \\
{\left[\times 10^{5}\right.} \\
\mathrm{cm} / \mathrm{s}]\end{array}$ & $\begin{array}{l}C_{t} \\
{\left[\times 10^{5}\right.} \\
\mathrm{cm} / \mathrm{s}]\end{array}$ & $R_{l}$ & $R_{f t}$ & Lit. \\
\hline $\mathrm{Sn}$ & 7.3 & 3.32 & 1.67 & 0.002 & 0.01 & {$[16]$} \\
$\mathrm{Al}$ & 2.7 & 6.26 & 3.08 & 0.03 & 0.01 & {$[16]$} \\
$\mathrm{In}$ & 7.28 & 2.51 & 1.30 & 0.04 & 0.0003 & {$[36]$} \\
$\mathrm{Konst.}$ & 8.8 & 5.24 & 2.64 & 0.06 & 0.15 & {$[16]$} \\
$\mathrm{Cr}$ & 7.19 & 6.85 & 3.98 & 0.04 & 0.20 & {$[37]$} \\
$\mathrm{N}_{2}$ & 1.026 & 1.77 & 0.88 & 0.75 & 0.69 & {$[38]$} \\
$\mathrm{Ne}$ & 1.51 & 1.06 & 0.81 & 0.87 & 0.60 & {$[39]$} \\
$\mathrm{Ar}$ & 1.677 & 1.51 & 0.81 & 0.69 & 0.57 & {$[40]$} \\
$\mathrm{He}$ & 0.145 & 0.23 & & 0.99 & 1.0 & {$[41]$} \\
\hline
\end{tabular}

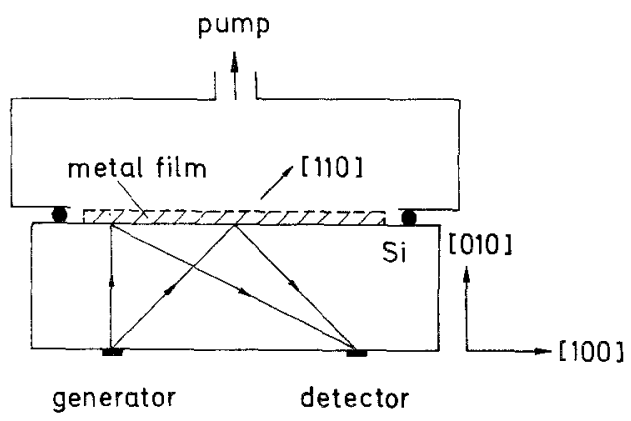

Fig. 5. Experimental set-up. The crystal is pressed with the scattering surface to a vacuum chamber. Generator and detector are in liquid helium

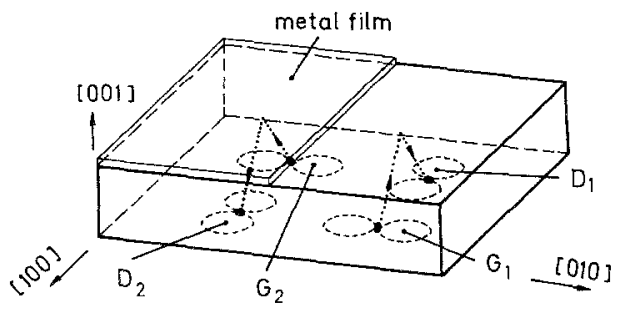

Fig. 6. Arrangement of the two generator/detector pairs on the substrate. Opposite to one pair $\left(G_{2} \rightarrow D_{2}\right)$ the scattering surface is covered with a metal film opposite to the other pair $\left(G_{1} \rightarrow D_{1}\right)$ the scattering surface is in vacuum

The reflection factors are measured by comparing the detector signals from the free and the covered surface of one crystal as shown in Fig. 6. In this arrangment the number of eventually different parameters, by using separate crystals for the measurement with and without covered surface as, e.g. crystal orientation, heater temperature, phonon transmission through tunneling junction/substrate - in- 
terface, quasiparticle lifetime, etc., is as small as possible.

Two independent generator/detector pairs are on the crystal. Opposite to one pair, the crystal surface is covered with the metal to be tested; opposite to the second pair, the crystal surface is uncovered. The two generators, respectively detectors, are simultaneously prepared. Because of the identical geometries, the parameters (heater resistance; energy gap; orientation; etc.) of the elements are largely the same. Only for tin-tunneling junctions different oxidebarriers are obtained, caused by the spacial inhomogeneity of the glow discharge in the evaporation apparatus. Hence, the thermal tunneling currents and the dynamic resistances differ up to $50 \%$. For the calculation of the reflection factors, we consider the individual sensitivity of the junctions by measuring the temperature dependence on the thermal current and the amplification factor (for details see [12]). We prove by control experiments that this method allows to measure the reflection factors $R_{\alpha}$ with a maximum error of about $10 \%$. The areas of the junctions are 0.15 and $0.28 \mathrm{~mm}^{2}$. To improve the signal-to-noise ratio, we use in some experiments elements with areas of 1 and $5 \mathrm{~mm}^{2}$. But in these measurements the time resolution is drastically reduced.

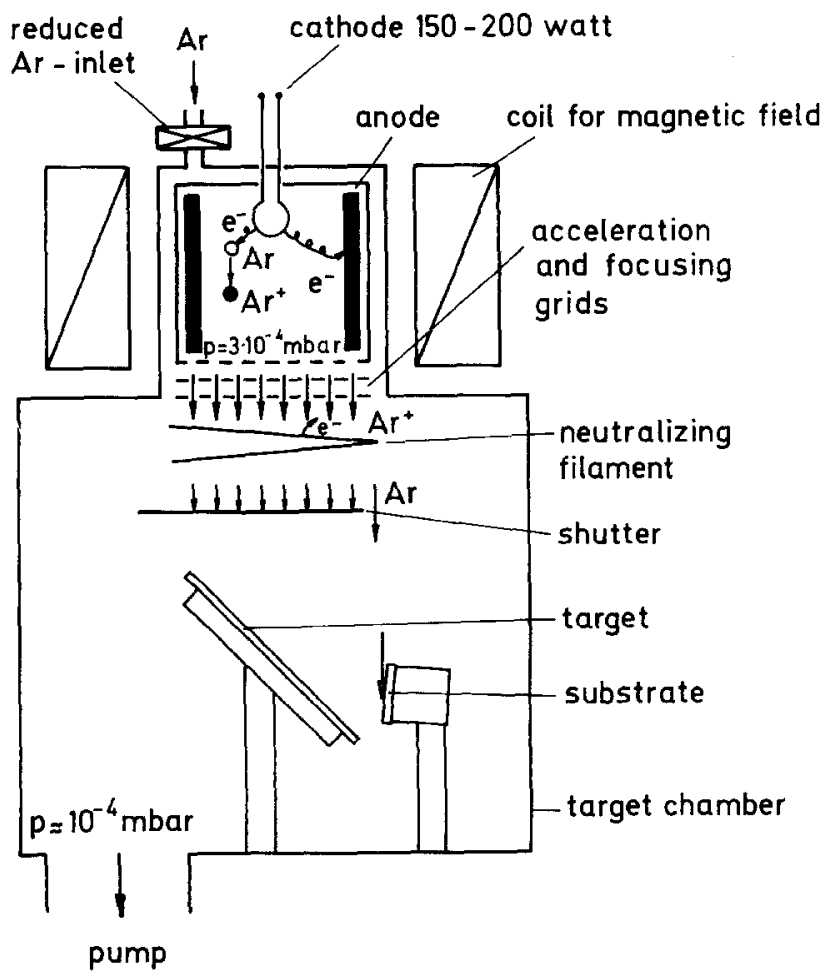

Fig. 7. Sketch of the sputter apparatus. With partially opened shutter the crystal surface can be sputter-cleaned. With completely opened shutter metal atoms are sputtered from the target to the substrate
After sawing and lapping the silicon crystals are mechanically polished with diamond grain, and the diameter stepwise decreased to $0.25 \mu \mathrm{m}$. Some crystals are, in addition, chemically etched (Schaeferetch) or DC-sputter-polished, using a Veeco microetch argon-ion gun (Ar-energy: 1,000 V-300 V; etch rate: $0.2-1 \mu \mathrm{m} / \mathrm{s}$ ). Chemical etching removes surface dislocations caused by mechanical polishing. The metal films are mostly prepared by thermal evaporation. After sputtering, the metal films are deposited without breaking the evaporator vacuum. Thus, the thickness of oxide and extraneous molecule layers in the interface is reduced, as compared with mechanical and chemical polishing.

Alternatively, the metal films are prepared by sputtering. Figure 7 shows the used arrangement which allows sputter cleaning of the substrate surface just before preparing the metal film. The experiments are performed using pulse technique to separate signal contributions by their different propagation time. A sample temperature of $1.4 \mathrm{~K}$ is used. At this temperature the effective quasiparticle lifetime in the detector is in the same order of magnitude as the time constant of the electronic equipment and, therefore, the compromise of time resolution and sensitivity reaches a maximum.

\section{Experimental Results and Discussion}

\subsection{Phonon Scattering at the Free Silicon Surface}

The typical detector signal, as shown in Fig. 8, exhibits four sharp pulses and a broad delayed background. The propagation times of the first and the third pulses agree with the expected time for specularly reflected longitudinal and fast transverse phonons respectively. Specularly reflected slow transverse phonons are defocused and cannot be detected. Remarkably, the propagation times of the second and the fourth pulses do not agree with the expected times for specularly reflected phonons. By covering selected parts of the scattering surface with an indium film, we are able to locate the areas from which the phonons of the fourth pulse are scattered (Fig. 9). The resulting propagation path indicates that the fourth pulse consists only of diffusively scattered phonons. Therefore, the simple view that a sharp pulse indicates specular reflection and a broad signal diffusive scattering, is misleading. Instead, phonon focusing in the silicon substrate forms narrow propagation channels where diffusively scattered phonons also reach the detector with little variation of their propagation times and give rise to a sharp pulse. 


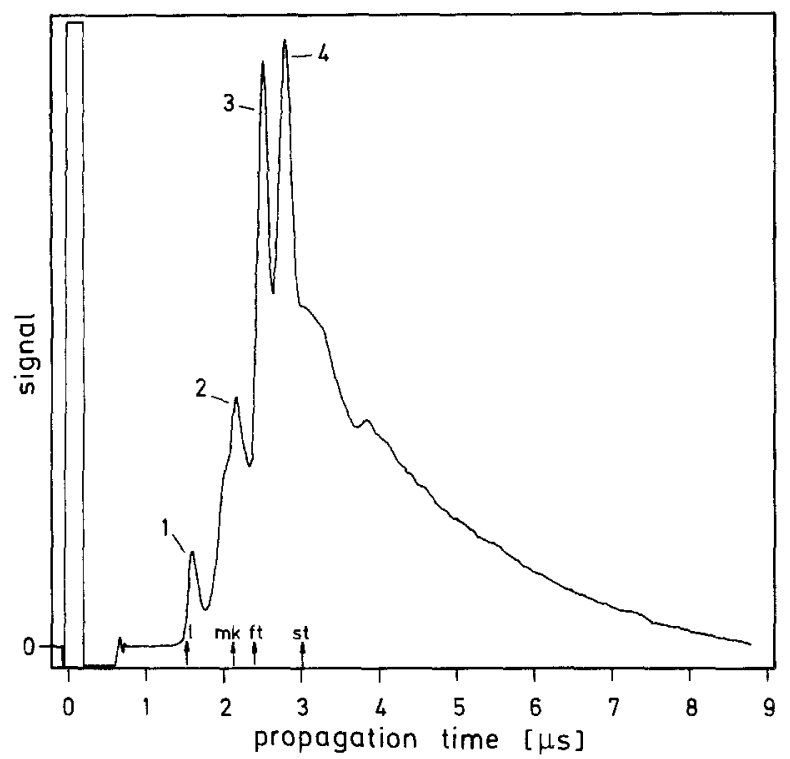

Fig. 8. Detector signal with high resolution. The generator/detector line forms an angle of about $4^{\circ}$ with the $|100|$-direction. Phonon-scattering at the free silicon surface. The calculated propagation times for specularly reflected phonons are marked by arrows. The pulses are numbered according to their propagation times. Heater power $3.6 \mathrm{~W} / \mathrm{mm}^{2}$, pulse duration $50 \mathrm{~ns}$, bath temperature $1.8 \mathrm{~K}$

Another effect of the phonon focusing is the strong influence of the exact position of the generator/detector elements on the detector signal. In the experiment (see Fig. 4) the generator-detector line forms an angle of appr. $4^{\circ}$ with the $|100|$ direction. When the generator-detector line is exactly parallel to the $|100|$ direction, one receives a detector signal, as shown in Fig. 10: the third pulse increases because of the phonon focusing so strongly that the fourth pulse can no longer be separated.

Since the fourth pulse is clearly due to diffusively scattered phonons, the phonons of the first and the third pulse can be either specularly reflected or diffusively scattered within additional propagation channels formed by phonon focusing. As the travelling times do not differ for both cases, other criteria are necessary to determine the amount of specular reflection. One possibility is the expected frequency dependence for diffuse scattering as compared to specular reflection. If diffusive scattering is caused by the roughness of the scattering surface, the contribution of the diffusively scattered phonons should increase with phonon frequency.

We analyse the frequency dependence of the detector signal qualitatively by using a constantan heater as generator and varying the heater power. Figure 11 shows detector signals for different heater powers and Fig. 12 the amplitude ratios as function of the heater power. Pulse 2 and Pulse 4 increase

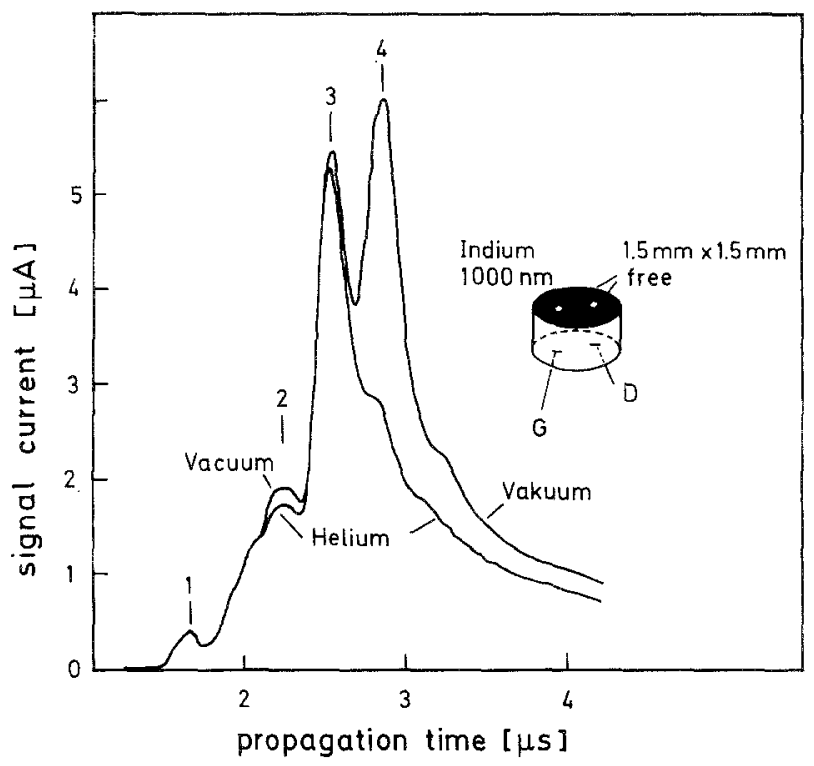

Fig. 9. Detector signal with indium-masked surface. Only the areas, on which the phonons of the fourth pulse are scattered, are free. Upper graph: surface in vacuum; lower graph: surface with additional helium cover. Heater power $4.5 \mathrm{~W} / \mathrm{mm}^{2}$, pulse duration $50 \mathrm{~ns}$; bath temperature $1.37 \mathrm{~K}$

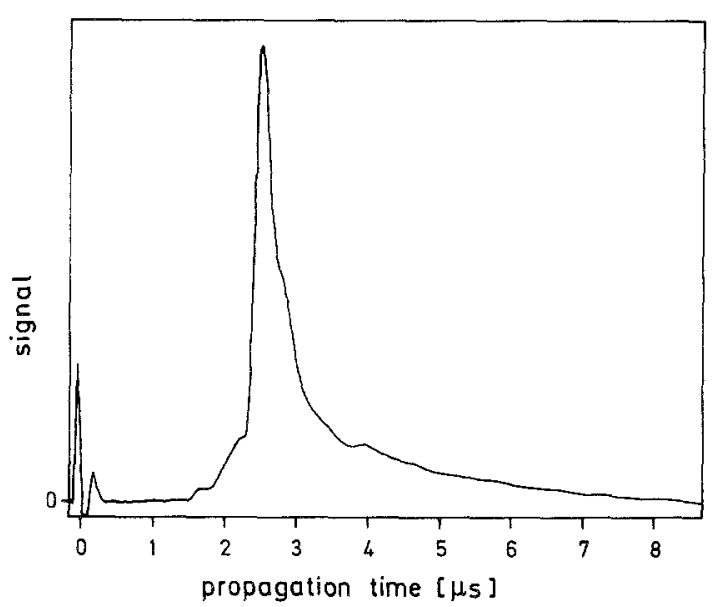

Fig. 10. Detector signal with an angle of about $0^{\circ}$ between generator/detector line and the $|100|$-direction. Phonons scattered at the free silicon surface. Heater power $1.7 \mathrm{~W} / \mathrm{mm}^{2}$; pulse duration $100 \mathrm{~ns}$; bath temperature $1.28 \mathrm{~K}$

with heater power significantly stronger than Pulse 1 and Pulse 3. Pulses 3 and 4 are caused by transverse phonons. Since the phonons of the fourth pulse, as discussed earlier, are diffusively scattered, we conclude from the results of Fig. 11 and 12 that a significant part of the phonons, causing the first and third pulses, are specularly reflected.

In order to check whether the observed frequency dependence may be caused by an inelastic surface 


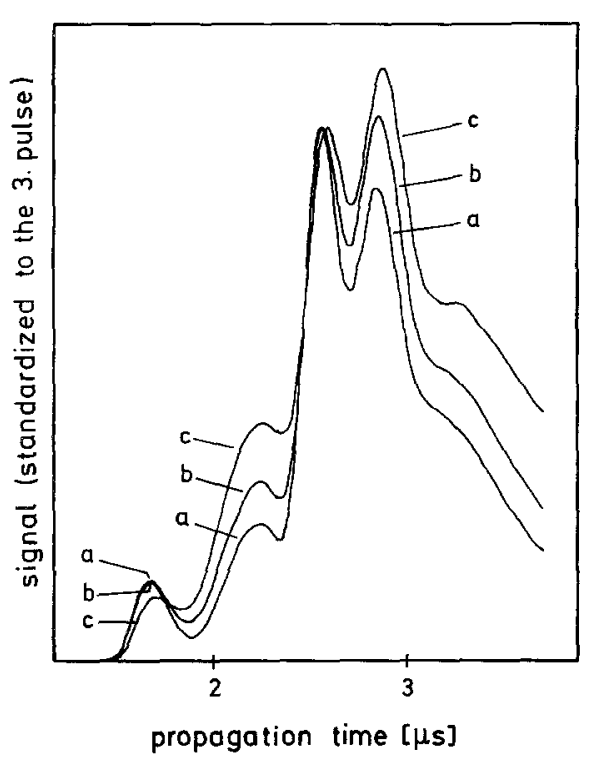

Fig. 11. Detector signal for different heater powers, standardized to the amplitude of the third pulse. Phonons scattered at the free silicon surface: a) heater power $44 \mathrm{~mW} / \mathrm{mm}^{2}$, gain $180 . b$ ) heater power $400 \mathrm{~mW} / \mathrm{mm}^{2}$, gain 18 c) heater power $4,400 \mathrm{~mW} / \mathrm{mm}^{2}$, gain 1. Pulse duration $100 \mathrm{~ns}$, bath temperature $1.39 \mathrm{~K}$

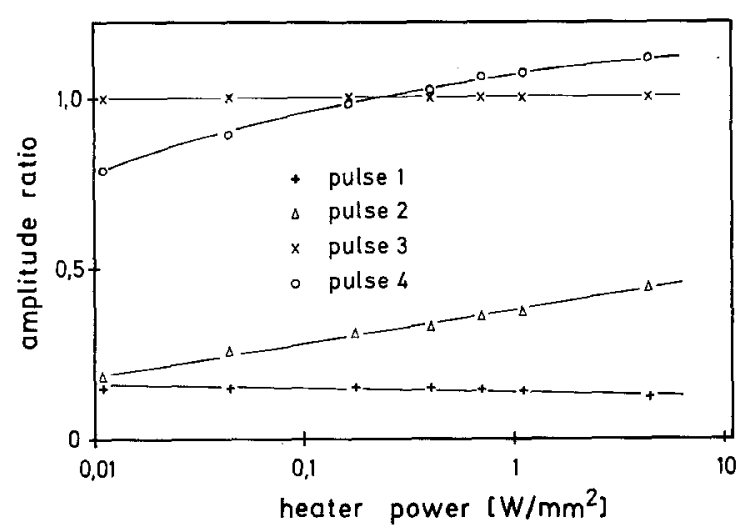

Fig. 12. Amplitudes of the single pulses divided by the amplitude of the third pulse in dependence on the heater power. Phonon scattered at the free silicon surface. Pulse duration $100 \mathrm{~ns}$, bath temperature $1.39 \mathrm{~K}$

decay of the phonons of the second and fourth pulses, we make experiments with superconducting tin tunneling junctions. In these experiments the phonon frequency is fixed at $280 \mathrm{GHz}$ and decayed phonons are not detected. The detector signals in these experiments do not show any significant difference to the signals measured with heaters as phonon generators (Fig. 13). Also the differentiated generatordetector characteristic (Fig. 14) verifies the energy $2 A_{\mathrm{Sn}}$ (see [11]) of the detected phonons.

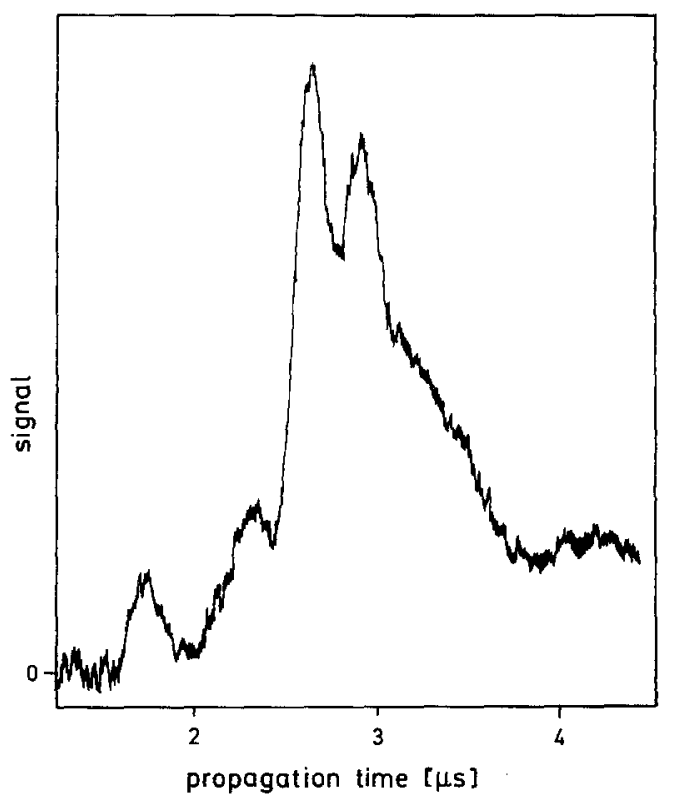

Fig. 13. Detector signal with Sn-tunneling junctions as generator and detector (monochromatic $280 \mathrm{GHz}$-phonons). Phonons scattered at the free silicon surface. Pulse duration $100 \mathrm{~ns}$, bath temperature $1.41 \mathrm{~K}$

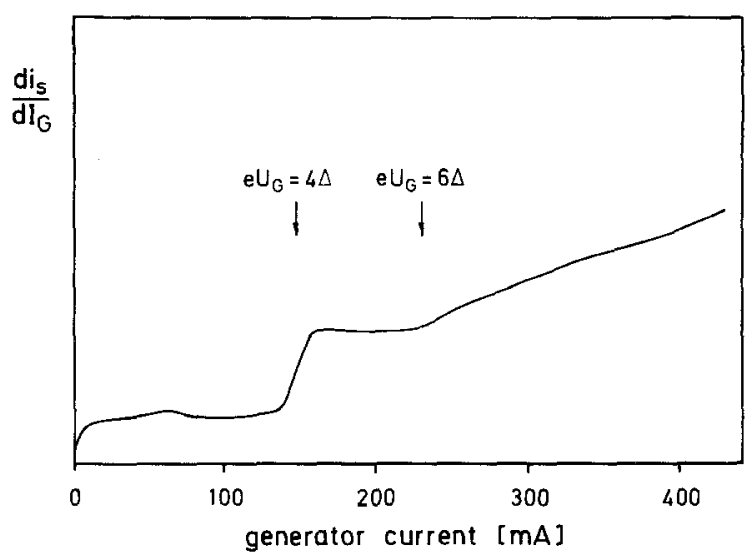

Fig. 14. Differentiated generator-detector characteristic with Sntunneling junctions as generator and detector. The step at $e U_{a}$ $=4 \Delta$ and the increase at $e U_{a}=6 \Delta$ indicate the detector of $2 \Delta$ phonons [11]. Phonons scattered at the free silicon surface

With superconducting tin tunneling junctions as generator and detector we measure phonon efficiencies (ratio of the observed signal amplitude divided by the theoretical signal amplitude) of $1.1 \%$ and $6 \%$ for longitudinal phonons. This is in accordance with earlier experiments by Trumpp and Eisenmenger [12] in phonon transmission with generator and detector exposed to liquid helium. Thus, no significant inelastic decay by phonon scattering at the free silicon surface is observed. In summary from our 
reflection experiments at the free silicon surface so far, the following tentative conclusions may be drawn:

- Diffusively scattered phonons give rise to sharp pulses and to a delayed background in the detector signal.

- The first and the third pulses differ in phonon frequency depence from the second and the fourth pulses. Obviously, this can be explained by the two scattering mechanisms differing in phonon frequency dependence. The most simple models are specular and diffusive scattering: An essential portion of specularly reflected phonons contributes to the first and third pulses. The second and the fourth pulses are caused by diffusively scattered phonons.

- Inelastic decay by scattering is negligible.

With these conclusions the experimental situation seems to be very satisfying. In a simple experiment one can study the behavior of specularly and diffusively scattered phonons by covering the free silicon surface with an arbitrary material. The change of the first and third pulses should be in accordance with the acoustic mismatch theory and the change of diffusively scattered phonons can be tested in observing the second and fourth pulses and the background.

\subsection{Phonon Scattering at Interfaces}

A number of material parameters may be relevant for the phonon scattering at interfaces:

- The acoustic impedances.

For ideal elastic interfaces the amount of backscattering is determined solely by the acoustic impedances. These may vary in a wide range: Condensed gases on silicon, due to the large mismatch, should lead to a reduction in backscattering of only $1 \%$ to $30 \%$ as compared to the backscattering from the free surface, whereas metals, being rather well-matched, should reduce the backscattering as much as $70 \%$ to $99 \%$.

- The phonon - electron-interaction.

The phonon absorption in the overlay may play a role for the backscattering. Since superconductors show a discontinuity of the absorption at the energy gap [28] we have used superconductors with different energy gaps and normal conducting metals.

- The chemical reactivity.

Aluminum reacts, for example, with the oxide covering the silicon crystal [29].

- The diffusion in silicon.

The average diffusion depth of $\mathrm{Cu}$ is about 8 orders of magnitude larger than of Sn [30].

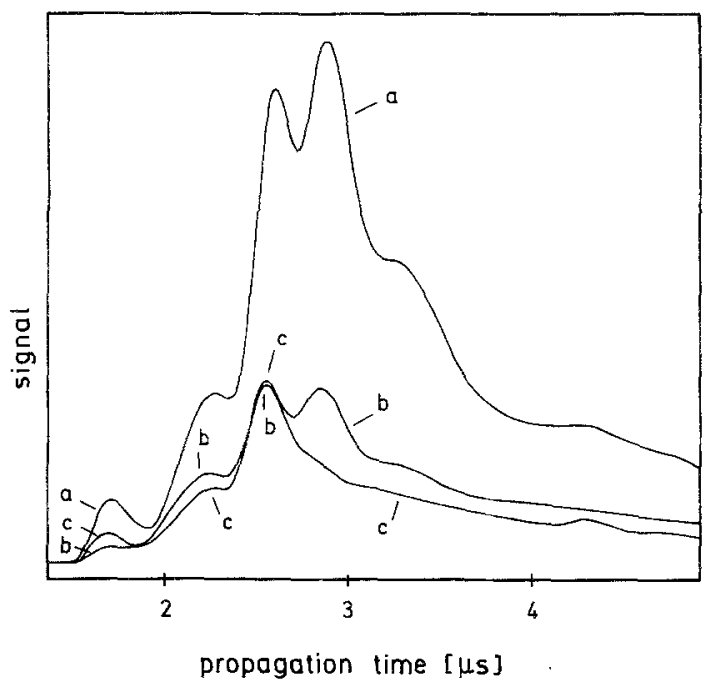

Fig. 15. Detector signal with scattering surface in vacuum $(a)$, covered with an $800 \mathrm{~nm}$ thick Sn-film (b) and with $\mathrm{Ne}(c)$

- The adhesion of the metal films.

Evaporated films of $\mathrm{Cr}$ and $\mathrm{Al}$ show very good adhesion. Also the preparation of the films and of the interfaces influences the adhesion.

- The formation of metal silicids.

$\mathrm{Cr}, \mathrm{Ni}$ form a silicid; $\mathrm{Al}, \mathrm{Sn}, \mathrm{Pb}$ do not [29].

We have chosen the substances to cover the surfaces under the aspect of varying these material parameters in a wide range. Finally, gases are condensed in two different ways on the scattering surface:

1) By gas inlet into the vacuum chamber cooled to $1 \mathrm{~K}$ and using a liquid nitrogen cold trap for purification.

2) By gas inlet into the $77 \mathrm{~K}$-cold vacuum chamber, the pressure at this temperature is below the condensation threshold. Condensation at the substrate surface is then obtained by cooling the sample to liquid helium temperature.

Method 2) reduces the signal amplitude of the backscattered phonons significantly stronger than Method 1), indicating that the insulator layer is denser in the preparation according to Method 2).

The metal films should be thick compared to the mean fee path of the phonons in the film. Only then the phonons scattered at the free film surface are not backscattered into the substrate. This condition is experimentally tested by covering the metal film with helium. With sufficiently thick metal film the helium does not significantly influence the signal of backscattered phonons. The corresponding minimal thickness of the films is found to be about $600 \mathrm{~nm}$ for Sn-films and appr. $100 \mathrm{~nm}$ for constantan films. Figure 15 shows the signals for backscattered phonons from the free silicon surface, as well as from 
Table 2. Calculated and measured values for the ratio $R_{\alpha}$. Above the measured values the preparation of the substrate surface ( $m p, c p$, ip: mechanical, chemical, sputter polish) and the thickness of the metal film are indicated

\begin{tabular}{|c|c|c|c|c|c|c|}
\hline \multirow{2}{*}{$\frac{\text { Material }}{\mathrm{Sn}}$} & \multirow[t]{2}{*}{ Calculated ratio $R_{\alpha}^{\text {theor }}$} & \multicolumn{5}{|c|}{ Measured ratio $R_{\alpha}^{\exp }$} \\
\hline & & $m p$ & $m p$ & $c p$ & ip & $c p, i p^{a}$ \\
\hline & & $400 \mathrm{~nm}$ & $630 \mathrm{~nm}$ & $1,000 \mathrm{~nm}$ & $1,000 \mathrm{~nm}$ & $>600 \mathrm{~nm}$ \\
\hline & $R_{l} \quad 0.002$ & 0.25 & 0.64 & 0.60 & 0.56 & 0.26 \\
\hline & $R_{2}$ no calc. value & 0.37 & $0.5 \mathrm{I}$ & 0.56 & 0.69 & 0.51 \\
\hline & $R_{f t} 0.01$ & 0.34 & 0.54 & 0.41 & 0.76 & 0.39 \\
\hline & $R_{4}$ no calc. value & 0.38 & 0.31 & 0.38 & 0.48 & 0.39 \\
\hline \multirow[t]{6}{*}{$\mathrm{Al}$} & & $m p$ & & & & \\
\hline & & $200 \mathrm{~nm}$ & & & & \\
\hline & $R_{1} \quad 0.03$ & 0.26 & & & & \\
\hline & $R_{2}$ no calc. value & 0.56 & & & & \\
\hline & $R_{f t} 0.01$ & 0.40 & & & & \\
\hline & $R_{4}$ no calc. value & 0.42 & & & & \\
\hline \multirow[t]{6}{*}{ In } & & $m p$ & $m p$ & $m p$ & & \\
\hline & & $800 \mathrm{~nm}$ & $1,600 \mathrm{~nm}$ & $1,100 \mathrm{~nm}$ & & \\
\hline & $R_{l} \quad 0.04$ & 0.41 & & & & \\
\hline & $R_{2}$ no calc. value & 0.38 & & & & \\
\hline & $R_{f t}^{2} 0.0003$ & 0.26 & $\sim 0.4$ & $\sim 0.43$ & & \\
\hline & $R_{4}$ no calc. value & 0.25 & & & & \\
\hline \multirow[t]{6}{*}{ Constantan } & & $m p$ & $m p^{b}$ & & & \\
\hline & & $100 \mathrm{~nm}$ & $145 \mathrm{~nm}$ & & & \\
\hline & $R_{l} \quad 0.06$ & 0.81 & 0.56 & & & \\
\hline & $R_{2}$ no calc. value & 0.63 & 0.66 & & & \\
\hline & $R_{f t} 0.15$ & 0.74 & 0.65 & & & \\
\hline & $R_{4}$ no calc. value & 0.42 & 0.34 & & & \\
\hline \multirow[t]{6}{*}{$\mathrm{Cr}$} & & $m p$ & & & & \\
\hline & & $280 \mathrm{~nm}$ & & & & \\
\hline & $R_{l} \quad 0.04$ & 0.41 & & & & \\
\hline & $R_{2}$ no calc. value & 0.81 & & & & \\
\hline & $R_{f t} 0.02$ & 0.78 & & & & \\
\hline & $R_{4}$ no calc. value & 0.42 & & & & \\
\hline \multirow[t]{5}{*}{$\mathrm{N}_{2}$} & & $m p$ & $i p$ & & & \\
\hline & $R_{l} \quad 0.75$ & & 0.47 & & & \\
\hline & $R_{2}$ no calc. value & & 0.46 & & & \\
\hline & $R_{f t} 0.69$ & $\sim 0.45$ & 0.47 & & & \\
\hline & $R_{4}$ no calc. value & & $<0.23$ & & & \\
\hline \multirow[t]{5}{*}{$\mathrm{Ne}$} & & $m p^{\mathrm{b}}$ & $m p$ & $i p$ & & \\
\hline & $R_{l} \quad 0.87$ & 0.63 & 0.43 & 0.45 & & \\
\hline & $R_{2}$ no calc. value & 0.50 & 0.49 & 0.44 & & \\
\hline & $R_{f t} 0.60$ & 0.47 & 0.39 & 0.40 & & \\
\hline & $R_{4}$ no calc. value & $<0.37$ & $<0.27$ & $<0.25$ & & \\
\hline \multirow[t]{5}{*}{$\mathrm{Ar}$} & & ip & & & & \\
\hline & $R_{l} \quad 0.69$ & 0.43 & & & & \\
\hline & $R_{2}$ no calc. value & 0.45 & & & & \\
\hline & $R_{f t} 0.57$ & 0.39 & & & & \\
\hline & $R_{4}$ no calc. value & $<0.25$ & & & & \\
\hline \multirow[t]{5}{*}{$\mathrm{He}$} & & $m p$ & $i p$ & & & \\
\hline & $R_{l} \quad 0.99$ & 0.67 & 0.70 & & & \\
\hline & $R_{2}$ no calc. value & 0.44 & 0.46 & & & \\
\hline & $R_{f t} 1.00$ & 0.40 & 0.42 & & & \\
\hline & $R_{4}$ no calc. value & $<0.20$ & $<0.20$ & & & \\
\hline
\end{tabular}

a Metal film sputter deposited by Ar-beam technique

b Generator was a tin tunneling junction 
the surface covered with $\mathrm{Sn}$ and with Ne. Table 2 contains the measured and calculated reflection factors.

We find, as a surprising experimental result, strong disagreement between the measured and the calculated reflection values. Materials expected to be acoustically well-matched to the silicon substrate show experimentally high phonon backscattering. Materials not expected to be well-coupled to the silicon show comparatively high phonon transmission.

The measured phonon backscattering of $30 \%$ to $60 \%$ is almost independent on the covering material and the preparation of the interface. The variation comes to about a factor 2 . This variation is small compared to the disagreement with the acoustic mismatch theory amounting roughly to a factor 10 to 1,000. Moreover, the different parts of the signal do not exhibit the behaviour which we expected according to the model of specular reflection of the first and third pulses and diffusive scattering of the second and fourth pulses.

Experimentally, we did not find any significant dependence of the reflection factors on the phonon frequency, i.e. by changing the heater power.

\subsection{Specular or Diffusive Scattering}

The experiments reported in the preceding chapter show that the scattering of phonons at "real" solid/ solid interfaces cannot be described by the acoustic mismatch model. Hence the well-known Kapitza anomaly at the solid/helium interface is only one aspect of the more general problem of deviations from the ideal acoustic transmission and reflection laws at interfaces. Because of the strong acoustic mismatch of a solid and helium, the failure of the acoustic mismatch theory is expecially evident for this system and was first found there.

Since our measurements did not indicate any significant dependence on the preparation of the interface and the special material forming the interface, we studied in more detail the phonon scattering at the most simple interface, i.e. the free surface.

As the acoustic mismatch theory predicts only specularly scattered phonons, it appears important to determine the real amount of specularly reflected phonons at a free silicon surface. For this purpose we compare the measured signal with signals calculated by a Monte Carlo method, for the cases of pure specular and pure diffusive scattering respectively.

For an elastically isotropic material and only one phonon polarization the calculated signals are pre-

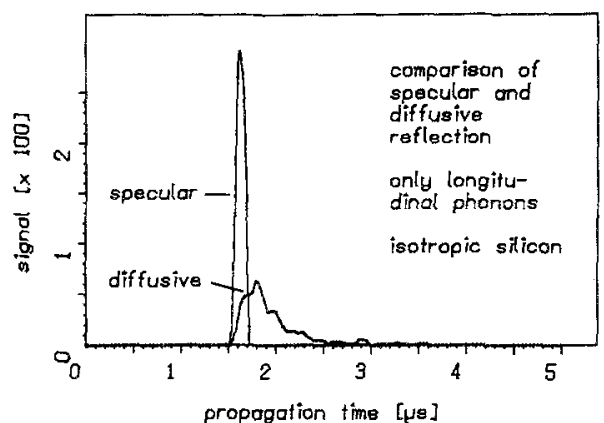

Fig. 16. Calculated detector signals for specular, respectively diffusive scattering from one phonon mode and elastically isotropic substrate. "Noise" of the diffusive signal is caused by statistical error of the Monte-Carlo-Calculation

sented in Fig. 16. As expected, specular reflection results in a narrow large pulse; diffusive scattering gives rise to a broad smeared signal. In contrast, diffuse scattering for the anisotropic silicon crystal with three phonon modes shows relatively narrow signals as consequence of phonon focusing. Therefore, the determination of the amount of specular and diffusive scattering requires a careful comparison of the calculated signal shapes and amplitudes with the experimental data.

Calculation of the Signal for Specular Reflection. The following assumptions are made to simplify the calculations:

- Besides the reduction of the number of longitudinal phonons by mode conversion, mode converted phonons are not considered.

- The particle density ratio for the different phonon modes emitted from the generator is given by the corresponding phonon density of states in the silicon. This is based on the non-ideal acoustic properties of the metal-insulator interface.

With these assumptions the calculated pulse amplitude ratios are still sufficiently accurate. We take fully into account the influence of the real geometric locations and dimensions of generator and detector, the generator pulse length and the phonon focusing on the pulse shape.

A calculated signal for pure specular reflection is shown in Fig. 17. It consists of three pulses; longitudinal, fast and slow transverse phonons. We calculate the signal for different angles between the generator/detector line and the $|100|$ direction of the crystal ("orientation"). Because of the phonon focusing, the amplitude ratios depend on this angle; with increasing angle the relative pulse amplitude of the fast transverse phonons decreases.

Calculation of the Signal for Diffusive Scattering. The problem of this calculation is how to distribute the 


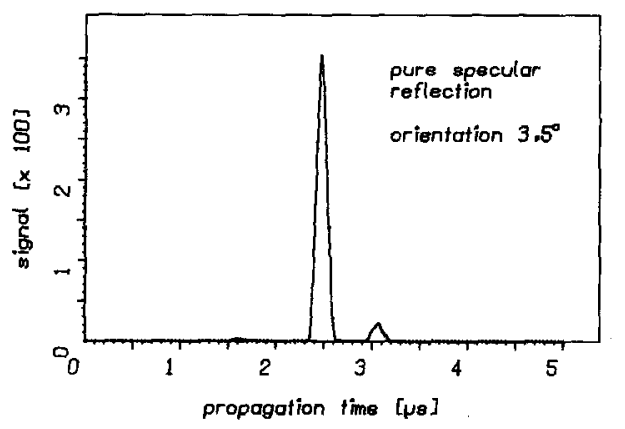

Fig. 17. Calculated detector signal for specularly reflected phonons without mode conversion. Duration of the generator pulse: $100 \mathrm{~ns}$

energy of the incident phonon on the scattered phonon modes. Since we do not know the microscopic scattering mechanism, we assume equal distribution for all momentum states in elastic scattering containing all possible propagation channels formed by the phonon focusing. This assumption corresponds to the condition of quasithermal equilibrium or detailed balance without changing the phonon energy. The angle distribution of the scattered phonons corresponds to Lambert's cosine law.

The calculated signal for pure diffusive scattering (Fig. 18) shows, indeed, a clear pulse structure indicating the existence of additional propagation channels caused by phonon focusing. The propagation times of the first four pulses agree well with the propagation times of the pulses in the measured signal. Again, the calculated signal depends strongly on the crystal orientation: the ratio of the amplitude of pulse four divided by the amplitude of pulse three increases with the angle between generator/detector line and the $|100|$ direction.

Surprisingly, the experimental signal, Fig. $15 \mathrm{a}$, can be described almost completely by the calculation for diffusive scattering, Fig. 18, alone. The pulse widths agree very well and the amplitude ratio for the third and fourth pulses of the measurement indicates less than $4 \%$ contribution by specular reflected phonons. Owing to this good agreement between measurement and calculation for pure diffusive scattering, we think a further refinement in fitting the calculation to the measurement would first require the improvement of the simple diffusive scattering model.

These results demonstrate, in contrast to the earlier discussion, that high-frequency phonon scattering at a "real", i.e. conventionally prepared silicon surface is almost completely diffuse.

To prove this further, we performed a reflection experiment with completely different orientations of generator and detector as shown in Figs. 19-21 to-

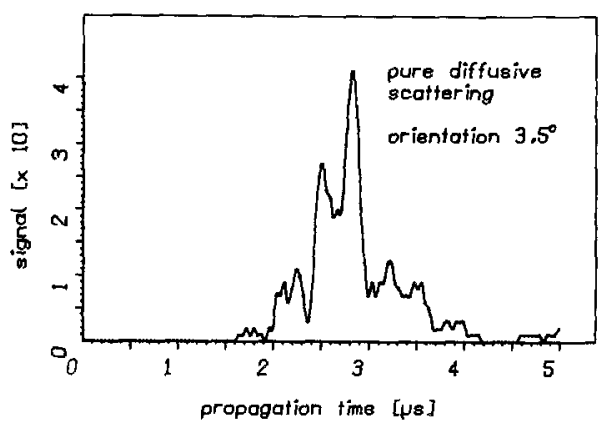

Fig. 18. Calculated detector signal for diffusively scattered phonons. Duration of the generator pulse: $100 \mathrm{~ns}$

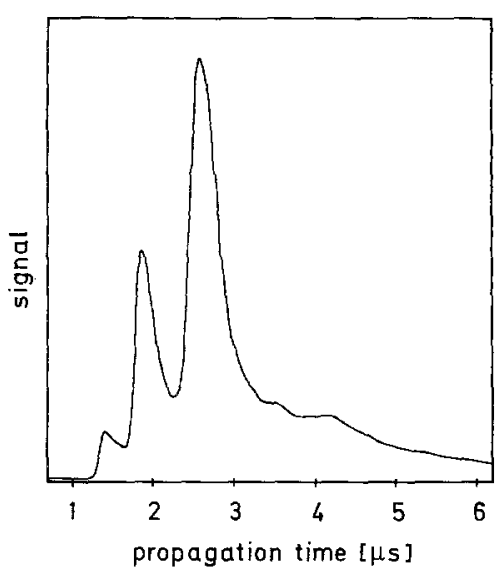

Fig. 19. Measured detector signal with generator and detector along the $|101|$-direction in a distance of $7.1 \mathrm{~mm}$. Pulse duration $100 \mathrm{~ns}$, bath temperature $1.28 \mathrm{~K}$

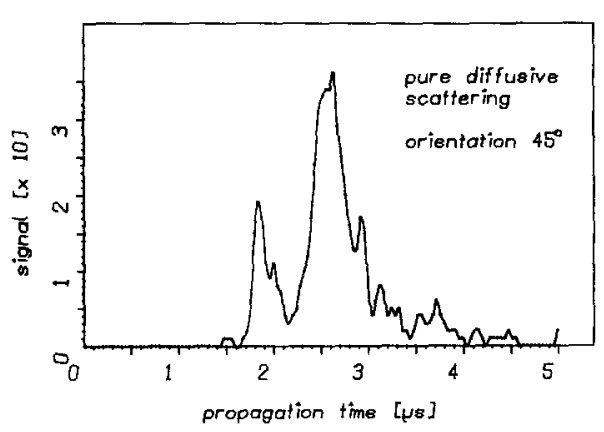

Fig. 20. Calculated detector signal for diffusively scattered phonons. Position of generator and detector according to the experiment of Fig. 19. Duration of the generator pulse: $100 \mathrm{~ns}$

gether with the measured and the calculated signals for pure diffusive and pure specular scattering. Again, the experimental result can be described convincingly by the calculation for diffusive scattering alone. 


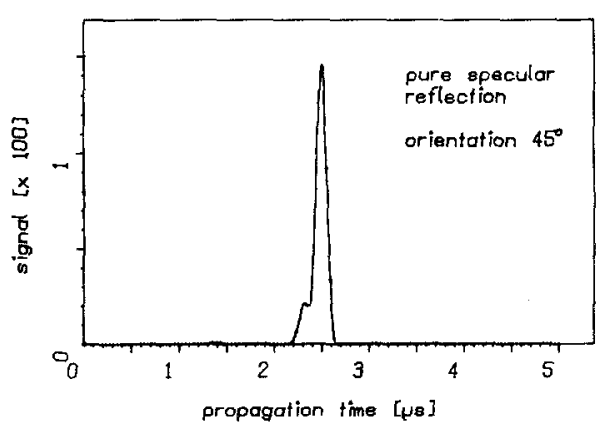

Fig. 21. Calculated detector signal for specularly scattered phonons. Position of generator and detector according to the experiment of Fig. 19. Duration of the generator pulse: $100 \mathrm{~ns}$

In another experiment we compared the signal of backscattered phonons from an optically rough surface with that from an optically very smooth surface. There is no significant difference between the corresponding signals, demonstrating that for short wavelength phonons the optically smooth surface is as rough as an optically rough surface.

With respect to the possible microscopic diffusive scattering mechanisms, so far, only limited experimental information is available. However, the amplitude ratios of the pulses and the change of these ratios with the phonon frequency, Fig. 12, allow a tentative discussion.

The stronger frequency dependence of the second and fourth scattering pulses, as compared to the first and third pulses, raises the question with respect to basic differences in the scattering conditions. The essential difference is the importance of mode conversion in scattering. For phonons of the first and third pulse, no mode conversion occurs, the polarization of the phonons remains unchanged. In contrast, the second pulse consists only of mode-converted phonons, whereas the fourth pulse implies mode conversion to a minor amount.

The absolute frequency dependence of the separate signal contributions indicates that at least two diffusive scattering mechanisms are involved. They differ in their dependence on the phonon frequency and in the probability of mode conversion.

The possible scattering mechanisms we propose are: 1) Diffusive elastic scattering caused by the geometric roughness of the surface with small mode conversion probability and weak frequency dependence.

2) Diffusive scattering caused by resonant scattering centers at the surface. The centers absorb the incident phonon and re-emit it with the same frequency (so-called two-level systems) but with high mode conversion probability and strong frequency dependence.
The scattering by two-level systems was suggested by Kinder [30] to explain the Kapitza anomaly. Corresponding to the properties of these models, we expect mechanism 1) to be dominant in the scattering of pulses 1 and 3 . With increasing phonon frequency, more and more phonons are scattered according to mechanism 2) and give rise to the measured stronger increase of the pulses two and four.

Checking the physical conditions for these scattering mechanisms, we found that by using a scanningelectron microscope our optically smooth surfaces exhibit scratches and dents of dimensions $\approx 50 \mathrm{~nm}$, in the same order of magnitude as the phonon wavelength. Hence, the condition for the scattering mechanism 1) is given. Calculations of the density of two-level systems, necessary to cause strong resonant phonon scattering $[31,32]$ show that less than one monolayer of two-level systems are sufficient. Also the increase of the scattering probability with the phonon frequency follows from these calculations [31].

The two-level systems are possibly caused by extraneous atoms absorbed at the surface or silicon atoms with special binding conditions at the disturbed surface. In these cases, we can certainly expect one monolayer of two-level systems at the surface far more than necessary to cause the observed diffusive scattering.

Since diffuse phonon scattering at the free silicon surface is not covered by the elementary acoustic mismatch theory, one cannot expect that the transmission of phonons through interfaces between silicon and other materials can be described by this theory. The phonon transmission through interfaces depends certainly on the microscopic scattering mechanism. But before making detailed considerations regarding the phonon transmission through interfaces one should first try to get more information on the scattering mechanisms.

Finally, it is important to note that, in contrast to the disagreement of our backscattering measurements and the acoustic mismatch theory, solid-solid heat transfer experiments $[6,4,5]$ show good agreement with the mismatch theory. The main differences of the heat transfer experiments and our experiments are:

- in heat transfer experiments, the phonons - transmitted through an interface - are in local thermal equilibrium. In our experiments, the phonons are far above the thermal equilibrium.

- in heat transfer experiments, only the phonon transmission summarized over all phonon modes, scattering angles and phonon energies is measured.

It is possible that the overall heat transport through an interface may approach the value predicted by 
the acoustic mismatch theory, although the single phonon is not scattered according to the mismatch theory.

Our calculations show that for the determination of the amount of diffusively scattered phonons it is necessary to consider the influence of the anisotropy of the substrate on the propagation time of the phonons, as well as on the pulse shape. It is not sufficient to take the anisotropy of the material into account only for the calculations of the propagation times and to calculate the pulse shape [33] solely in terms of an isotropic model.

\section{Conclusion}

The measured scattering of high-frequency acoustic phonons $(v \geqq 280 \mathrm{GHz})$ at solid/solid and solid/ vacuum interfaces cannot be described by the acoustic mismatch theory. Hence, the well-known deviation of the phonon scattering at solid/helium interfaces (Kapitza anomaly) from the mismatch theory is not a special case, as assumed till now. Because of the extreme acoustic mismatch of solids and helium the discrepancy in the theory was first found at the interface between these materials. On the other hand, two different solids in thermal contact show increased acoustic matching so that the deviation from the acoustic mismatch theory is not that obvious in heat transport experiments, integrating over all phonon modes, phonon frequencies and scattering angles.

We made sensitive reflection experiments with short phonon pulses, allowing separation of phonon frequencies, phonon modes and, to some extent, of scattering angles. We could clearly show that the phonons are not scattered according to the acoustic mismatch theory.

Nevertheless, for special combinations of material the thermal boundary resistance measured in heat conduction experiments can coincide with values expected by the acoustic mismatch theory. We found only a minor dependence of the measured phonon scattering on the materials forming the interface and the preparation procedure. Theoretically well-matched metals (expected reflection factor $\simeq 1 \%$ ) show increased reflection (measured reflection factor $\simeq 50 \%$ ); for mismatched condensed gases (expected reflection factor $\geqq 70 \%$ ) we measured decreased reflection (again $\simeq 50 \%$ ). Variation of the preparation of the substrate surface (mechanical, chemical and ion-polish) and of metal film (evaporation and sputtering) changes the phonon reflec- tion about a factor 2 . This change is small compared with the deviation from the acoustic mismatch theory.

The analysis of the signal of scattered phonons is complicated by the anisotropy for phonon propagation of the substrate (phonon focusing). Comparison of the measured signal at a free silicon surface with calculated signals, assuming pure specular and pure diffusive scattering respectively, show that only diffusive scattering is present. The upper limit for specularly reflected contributions is about $4 \%$. For the first time in these calculations the influence of the anisotropy of the substrate and the dimensions and locations of generator and detector to the pulse shape are fully taken into account.

Within the accuracy of our measurements we did not find any significant phonon decay by anharmonic processes at the uncovered surface.

The signal amplitude of backscattered phonons changes with the phonon frequency. We suggest to explain this phonon frequency dependence by two different microscopic, diffusive scattering mechanisms:

- Geometrical diffusive scattering caused by the roughness of the substrate surface;

- Resonant diffusive scattering caused by two-level systems at the surface.

In the frequency range of our experiment the number of phonons, being resonantly scattered by twolevel systems, increases with phonon frequency.

At solid/helium interfaces prepared by cleaving at $4 \mathrm{~K}$, Weber et al. [34] measured phonon scattering according to the acoustic mismatch theory. But contamination by gas molecules or cleavage steps cause, again, large deviations from the theory. Therefore, evaporation of metals or insulators even on ideal cleavage surfaces may create scattering centers causing again strong diffuse scattering.

A method to prepare an interface with higher specularly scattered contributions may be molecular beam epitaxy [35]. Improved interfaces prepared by this technique are important for investigating microscopic scattering mechanisms.

A more general technique for the preparation of "ideal" insulator-metal interfaces is important for achieving higher resolution and sensitivity in phonon experiments with superconducting tunneling junctions.

The authors gratefully acknowledge financial support by the Deutsche Forschungsgemeinschaft and valuable discussions with S. Döttinger, O. Koblinger, K. Laßman and M. Welte. We would like to thank Mrs G. Mayerhöffer for preparing the substrates. 


\section{References}

1. Kapitza, P.L.: J. Phys. (USSR) 4, 181 (1941)

2. Little, W.A.: Can. J. Phys. 37, 334 (1959)

3. Khalatnikov, I.M.: Zh. Eksp. Theor. Fiz. (USSR) 22, 687 (1952)

4. Peterson, R.E., Anderson, A.C.: J. Low Temp. Phys. 11, 639 (1973)

5. Anderson, A.C.: In: Nonequilibrium superconductivity, phonons and Kapitza boundaries. Gray, K.E. (ed.). New York: Plenum Press 1981

6. Nitsche, F., Schumann, B.: J. Low Temp. Phys. 39, 119 (1980)

7. Herth, P., Weis, O.: Z. Angew. Phys. 29, 101 (1970)

8. Kappus, W., Weis, O.: J. Appl. Phys. 44, 1947 (1973)

9. Challis, L.J.; J. Phys. C 7, 481 (1974)

10. Wyatt, A.F.G.: In: Nonequilibrium superconductivity, phonons and Kapitza boundaries. Gray, K.E. (ed.). New York: Plenum Press 1981

11. Eisenmenger, W.: In: Physical acoustics. Mason, W.P. (ed.), Vol. XII. New York: Academic Press 1976

12. Trumpp, H.J., Eisenmenger, W.: Z. Phys. B - Condensed Matter 28, 159 (1977)

13. Trumpp, H.J., Laßmann, K., Eisenmenger, W.: Phys. Lett. 41 A, 431 (1972)

14. Auld, B.A.: Acoustic waves in solids. New York, London: John Wiley 1973

15. Elbaum, C.: Int. Conf. on Phonon Scattering in Solids. Albany, H.J. (ed.) Paris 1972

16. Rösch, F., Weis, O.: Z. Phys. B - Condensed Matter 27, 33 (1977)

17. Every, A.G.: Phys. Rev. Lett. 42, 1065 (1979)

1.8. Eisenmenger, W.: In: Phonon scattering in condensed matter. Maris, H.J. (ed.) New York, London: Plenum Publishing Corp. 1980

19. Northrop, G.A., Wolfe, J.P.: In: Phonon scattering in condensed matter. Maris, H.J. (ed.) New York, London: Plenum Publishing Corp. 1980

20. Steinbrïchel, Ch.: Z. Phys. B - Condensed Matter 24, 293 (1976)

21. Weis, O.: Z. Angew. Phys. 26, 325 (1969)

22. Kinder, H.: Phys. Rev. Lett. 28, 1564 (1972)

23. Forkel, W., Welte, M., Eisenmenger, W.: Phys. Rev. Lett. 31, $215(1973)$
24. Forkel, W.: (to be published)

25. Marx, D., Buck, J., Laßmann, K., Eisenmenger, W.: J. Phys. (Paris) C6, Suppl. to No. 8, C6-1015 (1978)

26. Marx, D., Eisenmenger, W.: Phys. Lett. 82 A, 291 (1981)

27. Marx, D., Eisenmenger, W.: (to be published)

28. Bobetic, U.M.: Phys. Rev. 136 A, 1535 (1964)

29. Tu, K.N., Mayer, J.W.: IBM Research Report RC6349 (1977)

30. Sharma, B.L.: Diffusion in semiconductors. Clausthal-Zellerfeld: Trans. Techn. Publication 1970

31. Kinder, H.: Proc. 16th Int. Conf. on Low Temp. Phys. p. 549 (1981)

32. Arnold, W., Hunklinger, S., Dransfeld, K.: Phys. Rev. B 19, $6049(1979)$

33. Taborek, P., Goodstein, D.L.: Phys. Rev. B 22, 1550 (1980)

34. Weber, J., Sandmann, W., Dietsche, W., Kinder, H.: Phys. Rev. Lett. 40, 1469 (1978)

35. Narayanamurti, V.: In: Nonequilibrium superconductivity, phonons and Kapitza boundaries. Gray, K.E. (ed.). New York: Plenum Press 1981

36. Aleksandrov, K.S., Gurovits, L.S., Kamenskii, E.I.: Sov. Phys. Acoustics 6, 170 (1961)

37. Schramm, K.-H.: Z. Metallkd. 53, 729 (1962)

38. Benylyi, P.A., Tarasenko, L.M.: Sov. Phys. Solid State 10, $1660(1969)$

39. Leake, J.A., Daniels, W.B., Skalyo, J., Frazer, B.C.: Phys. Rev. 181, 1251 (1969)

40. Barker, J.R., Dobbs, E.R.: Philos. Mag. 46, 1069 (1955)

41. Wilks, J.: The properties of liquid and solid helium. New York: Clarendon Press 1967

D. Matx

Porsche AG

D-7251 Weissach

Federal Republic of Germany

W. Eisenmenger

Physikalisches Institut

Universität Stuttgart

Pfaffenwaldining 57

D-7000 Stuttgart 80

Federal Republic of Germany 